\title{
Hurdles to clinical translation of human induced pluripotent stem cells
}

\author{
Evgenios Neofytou, ${ }^{1,2,3}$ Connor Galen $\mathbf{O}^{\prime}$ Brien, ${ }^{1,3}$ Larry A. Couture, ${ }^{4}$ and Joseph C. Wu $\mathbf{u}^{1,2,3}$ \\ ${ }^{1}$ Stanford Cardiovascular Institute, ${ }^{2}$ Institute of Stem Cell Biology and Regenerative Medicine, and ${ }^{3}$ Department of Medicine, Division of Cardiology, Stanford University School of Medicine, Stanford, \\ California, USA. ${ }^{4}$ Beckman Research Institute of City of Hope, Duarte, California, USA
}

\begin{abstract}
Human pluripotent stem cells are known to have the capacity to renew indefinitely, being intrinsically able to differentiate into many different cell types. These characteristics have generated tremendous enthusiasm about the potential applications of these cells in regenerative medicine. However, major challenges remain with the development and testing of novel experimental stem cell therapeutics in the field. In this Review, we focus on the nature of the preclinical challenges and discuss potential solutions that could help overcome them. Furthermore, we discuss the use of allogeneic versus autologous stem cell products, including a review of their respective advantages and disadvantages, major clinical requirements, quality standards, time lines, and costs of clinical grade development.
\end{abstract}

\section{Introduction}

Over the last decade, regenerative medicine has been hailed as a "game changer" in modern medicine. Stem cells can be used to repair or replace damaged tissues in the human body by either promoting endogenous regenerative processes or directly replacing damaged tissues after cellular transplantation (1). Since the advent of human embryonic stem cells (ESCs) in 1998 (2) and the identification of their ability to self-renew indefinitely in vitro and differentiate into all three germ layers (ectoderm, mesoderm, and endoderm), the stem cell research community has identified ever more suitable tissue sources for exploring cell therapy and endogenous repair in humans. However, there are two significant obstacles associated with ESCs that hinder progress and clinical translation of such therapies: (a) ethical concerns because these cells are isolated from the inner cell mass of the human embryo (3) and (b) immune rejection problems because these cells are isolated from an allogeneic source (4).

In 2006 and 2007, Takahashi and Yamanaka made landmark discoveries in mouse and human induced pluripotent stem cells (iPSCs), respectively, with the introduction of only four transcription factors, namely OCT4, SOX2, KLF4, and c-MYC $(5,6)$. This approach circumvented the usual ethical problems associated with ESCs and raised the possibility of autologous transplantation. The discovery of iPSCs led to many more studies in the pluripotent arena, including developing "disease-in-a-dish" models for drug-screening platforms, generating disease-specific iPSC lines to study the pathophysiology of diseases, and creating personalized therapies for autologous stem cell transplantation (7).

In 2010, Geron Corporation began a stem cell clinical trial in patients with spinal cord injuries that was halted a year later due to changes in the business strategy of the company $(8,9)$. In 2014 , a new wave of first-in-human clinical studies was initiated. These

Conflict of interest: J.C. Wu is a cofounder of Stem Cell Theranostics.

Reference information: / Clin Invest. 2015;125(7):2551-2557. doi:10.1172/JCI80575. studies use pluripotent stem cell (PSC) sources (defined as both ESC and iPSC derivatives) to treat patients with spinal cord injuries (9), age-related macular degeneration (10-12), and type 1 diabetes (13). PSC-based products for the treatment of Parkinson's disease (14), heart failure (14), and several others are currently in the pipeline (15). Despite the vast potential of these PSC sources, the risks-versus-benefits analysis for such cell therapies is not clear-cut, given that there are still key limitations that continue to complicate their clinical translation. It is important to recognize that, because stem cell product derivatives represent an entirely novel treatment approach, the clinical translation of such experimental therapies may be correspondingly more complex and time consuming.

In this Review article, we evaluate the technical and practical obstacles to the clinical translation of these PSC derivatives and possible solutions that can bring personalized or precision medicine closer to reality. We also discuss preclinical challenges that must be addressed, including inherent tumorigenic potential of PSCs due to their properties of self-renewal and pluripotency and problems arising from their differentiation into heterogeneous mature adult types as well as issues with immunogenicity (16), engraftment, and survival. In the latter part of the article, we discuss some of the considerations, steps, and standards that need to be implemented for autologous and/or allogeneic iPSC use. We focus on preparations needed for cell bank setup and scalable PSC-derived product manufacturing that will be necessary to establish effective clinical implementation and realize the full potential of these novel therapies (Table 1).

\section{Early implementation of good manufacturing practices-compliant cell production}

As stem cell technology becomes a reality, one major goal is the establishment of the best methods to develop tissues for clinical application. Effective planning for commercialization requires anticipation of clinical requirements, production demands, and the resultant costs. Advance planning is essential for stem cell technologies because of their time intensive nature and high development costs. PSCs, such as ESCs or iPSCs, are highly versatile and read- 
Table 1. Translating human PSCs to therapies

\begin{tabular}{|c|c|c|c|c|c|}
\hline Characterization assays & $\begin{array}{l}\text { Master cell bank } \\
\text { development }\end{array}$ & $\begin{array}{c}\text { Safety } \\
\text { considerations }\end{array}$ & Product development & Potency assays & Clinical development \\
\hline Cell line stability in vitro & cGMP controlled & $\begin{array}{l}\text { Tumorigenicity/ } \\
\text { teratoma formation }\end{array}$ & $\begin{array}{l}\text { Cell line development/master cell } \\
\text { bank }\end{array}$ & Endpoint readout development & Preclinical studies in animal models \\
\hline Karyotyping & Pathogen free & $\begin{array}{c}\text { Stability of } \\
\text { differentiated } \\
\text { phenotype in vivo }\end{array}$ & $\begin{array}{l}\text { Process development (raw materials, } \\
\text { cellular impurities, cryopreservation, } \\
\text { supply chain, scalable cell culture) }\end{array}$ & Mechanism of action & IND with FDA \\
\hline Differentiation capacity & $\begin{array}{l}\text { Consent for iPSC } \\
\text { generation }\end{array}$ & $\begin{array}{l}\text { Dedifferentiated } \\
\text { potential }\end{array}$ & Commercial production & $\begin{array}{l}\text { Measuring the "active" } \\
\text { constituents }\end{array}$ & Phase I clinical studies \\
\hline $\begin{array}{l}\text { Expression of pluripotency } \\
\text { antigens }\end{array}$ & $\begin{array}{l}\text { Medical history } \\
\text { documentation }\end{array}$ & & & Use of the reference standard & Phase II clinical studies \\
\hline Purity assays & & & & Potency ratio & Phase III clinical studies \\
\hline \multirow[t]{2}{*}{ Level of cell type heterogeneity } & & & & Cell dosage identification & Register medical product with FDA \\
\hline & & & & & Clinical practice \\
\hline
\end{tabular}

The major areas that a company or a scientific institution needs to address in order to achieve the development and clinical translation of stem cell medicinal products.

ily produced in very large numbers. These valuable features make them ideal starting materials for developing scalable commercial cell products (17). However, manufacturing clinical-grade stem cell products for a clinical trial presents a number of difficult challenges that are not present in a research setting. For instance, reproducible manual handling in good manufacturing practice (GMP) clean room facilities needs to be established throughout the product's life cycle (18-20). For the best outcome, GMPs should be instituted as early as possible in the process. Doing so can avoid additional problems while ensuring that the manufacturing process complies with quality control standards of regulatory agencies to create a product that is cost effective, robust, scalable, safe, and reproducible, with the maximum chance of achieving commercial success.

With PSCs, the use of defined cultured systems should be implemented $(21,22)$. It is preferable to avoid using chemically undefined media or materials of animal origin, such as fetal bovine serum and mouse embryonic fibroblasts, as support systems, because they carry a risk of transmitting xenopathogens to the recipient patients. Rigorous quality controls, documentation, and adherence to current GMP (cGMP) for each manufactured cell batch are essential. Furthermore, it is crucial to ensure that products are consistently manufactured and meet all necessary criteria in terms of viability, function, purity, and sterility during the differentiation. Finally, once the required specifications are confirmed, certificates of analysis should be generated for all product lots to certify them for clinical use (Figure 1).

\section{Biodistribution testing of clinical target materials}

For preclinical animal studies, it is crucial that the PSC products studied are manufactured using processes comparable to those intended for the final GMP product. This is an important step, because these studies may be used to support future investigational new drug (IND) applications filed with the FDA. Although robust differentiation efficiencies can be achieved, undifferentiated cells could still remain within the cell product. Preclinical studies are intended to assess product safety, off-target effects, and the potential for teratoma formation from undifferentiated cells within the transplanted cell product.
Traditional histopathological techniques for preclinical studies cannot pinpoint the underlying mechanisms of stem cell biodistribution, engraftment, and migration in real time. Therefore, the risk of ectopic engraftment is unclear. It is also unknown where the nonengrafted cells go immediately after transplantation and what hazards they might present. Recent advances in positron emission tomography (PET) imaging, magnetic resonance imaging (MRI), fluorescence imaging (FLI), bioluminescence imaging (BLI), and other techniques have improved monitoring of transplanted grafts tremendously $(23,24)$. This has led to great progress in understanding cell behavior in vivo within tissues of interest, enabling the spatiotemporal mapping of transplanted cells for both long-term and short-term safety studies required by the FDA. For example, both BLI and MRI have been used successfully in small and large animal studies to track reporter gene or iron-labeled cells for long periods of time $(25,26)$. Although some of the aforementioned technologies have not yet become common in preclinical or clinical studies or are unlikely to do so because they are more suited for small animal work (e.g., FLI and BLI), such technologies are of growing importance and have already been used to study microenvironmental factors that regulate cell fate decisions (e.g., physical contact, cell-to-cell interactions, and adhesive properties of the cells in their interactions with the native tissue).

\section{Tumorigenicity studies}

The most significant posttransplantation safety concern is the formation of either benign teratoma or malignant teratocarcinoma $(27,28)$. Because stem cell-derived clinical products may consist of a heterogeneous population of cells, it is crucial to avoid inadvertently introducing neoplasms. Heterogeneity seen during the differentiation process usually is a result of the product being contaminated with undifferentiated cells or the use of a differentiation process that yields cells of multiple lineages. In addition, the cell product might dedifferentiate into cells capable of producing neoplasms. Karyotype stability studies of the undifferentiated starting material and efforts to remove residual undifferentiated cells are necessary to ensure that the transplanted product presents a minimal tumor risk. 


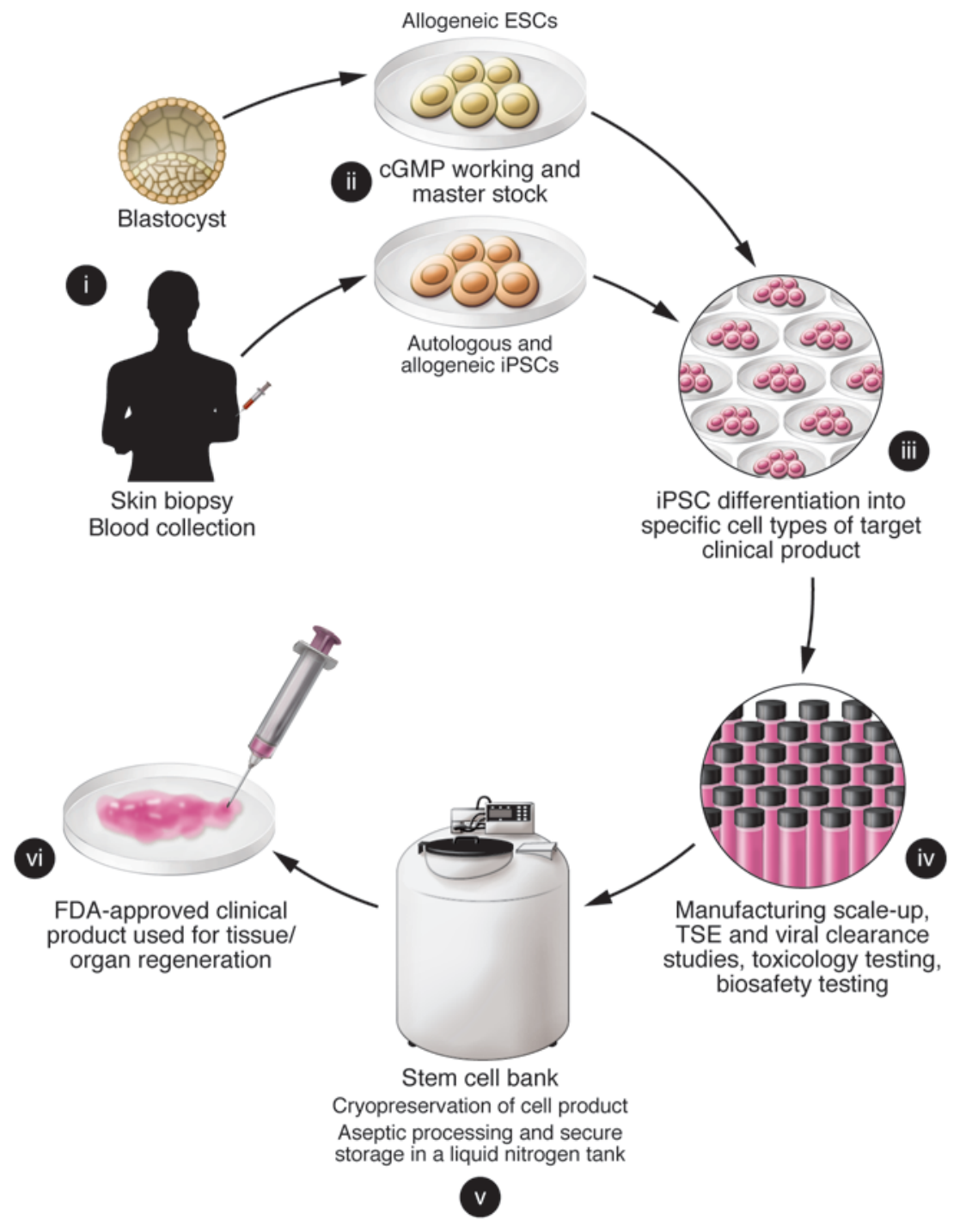

Figure 1. Flow chart of pluripotent stem cell banking. Human PSCs are ideal candidates for developing cell therapies from either a tissue biopsy or blood collection (i). Allogeneic and autologous stem cell banking requires establishment and expansion of the starting pluripotent material (ii). Human ESCs and iPSCs are capable of differentiating into any adult cell type (iii). Therapeutically relevant cell products must undergo preclinical studies required by the FDA prior to an IND application (iv). TSE, transmissible spongiform encephalopathy. Cells must undergo processing prior to secure cryostorage (v). The cryopreserved products may either be used immediately or be held for future use as allografts in order to regenerate damaged organs (vi).

\section{Toxicity studies}

The FDA requires that all stem cell clinical products undergo safety/toxicity studies before obtaining IND approval (36). These studies are typically designed to demonstrate that stem cell derivatives are nontoxic and otherwise safe in preclinical animal models. The models used depend on the study and indication of product use. Preclinical studies are usually done in normal animals in acute, subacute, and chronic models for varying periods varying from 3, 9, 12, and 24 months. For these studies, the FDA requires investigators to look for major organ toxicity and blood counts of animals after cell transplantation. In addition, cell products must be tested rigorously for acute infusion toxicity that might result from damage to the site of

Following cell transplantation, the toxicity and tumorigenicity of these cells can also be monitored by histologic assessment or human Alu quantitative PCR analysis $(29,30)$. Although human Alu quantitative PCR assay sensitivity is up to $0.1 \%$ compared to host genomic DNA, even greater sensitivity is desired. Newer methods such as digital PCR can improve the sensitivity and are currently being tested. Furthermore, strategies for tumor surveillance now exist that can provide cell death on demand, which are attractive fail-safe options to deal with possible tumor formation (31). For example, PET reporter genes, such as herpes simplex virus truncated thymidine kinase (HSV-tk), can be used for tumor surveillance because they allow the transplanted cells to take up substrates for imaging studies. In addition, these reporters act as suicide genes for tumor ablation therapy by inducing sensitivity to antiviral drugs such as ganciclovir (32, 33). In addition, several small molecules have been identified to eliminate human PSCs and prevent teratoma formation; use of these compounds may soon increase the safety profile of stem cell-based treatments $(34,35)$. Ultimately, only long-term studies of the transplanted cell products will be able to accurately assess the tumorigenesis risk associated with such therapies. transplantation as well as collateral damage to adjacent tissues stemming from an immune response against the cell product.

\section{Immunogenicity studies}

Poor stem cell survival and engraftment after delivery is partly due to cellular rejection triggered by the host's immune response (37). The lack of an effective method of inducing immune tolerance to maintain graft survival is a major roadblock for cell-based therapies. PSCs were once considered immune privileged due to their lack of MHC class I, MHC class II, and costimulatory molecules (38). Although undifferentiated ESCs might be immune privileged, it has been shown that their differentiated derivatives can trigger cellular and humoral immune responses (39). By contrast, autologous iPSCs may avoid the costs and side effects associated with lifelong immunosuppression required for allogeneic cell transplantation (16). Despite some controversy over the immunogenicity of undifferentiated iPSCs (40), recent work has demonstrated that the differentiation of iPSCs results in the loss of immunogenicity (41). Pending further validation, this is a potentially important finding, because it could lead to induction of tolerance similar to the tolerance elicited by a corresponding self-somatic cell. 
A
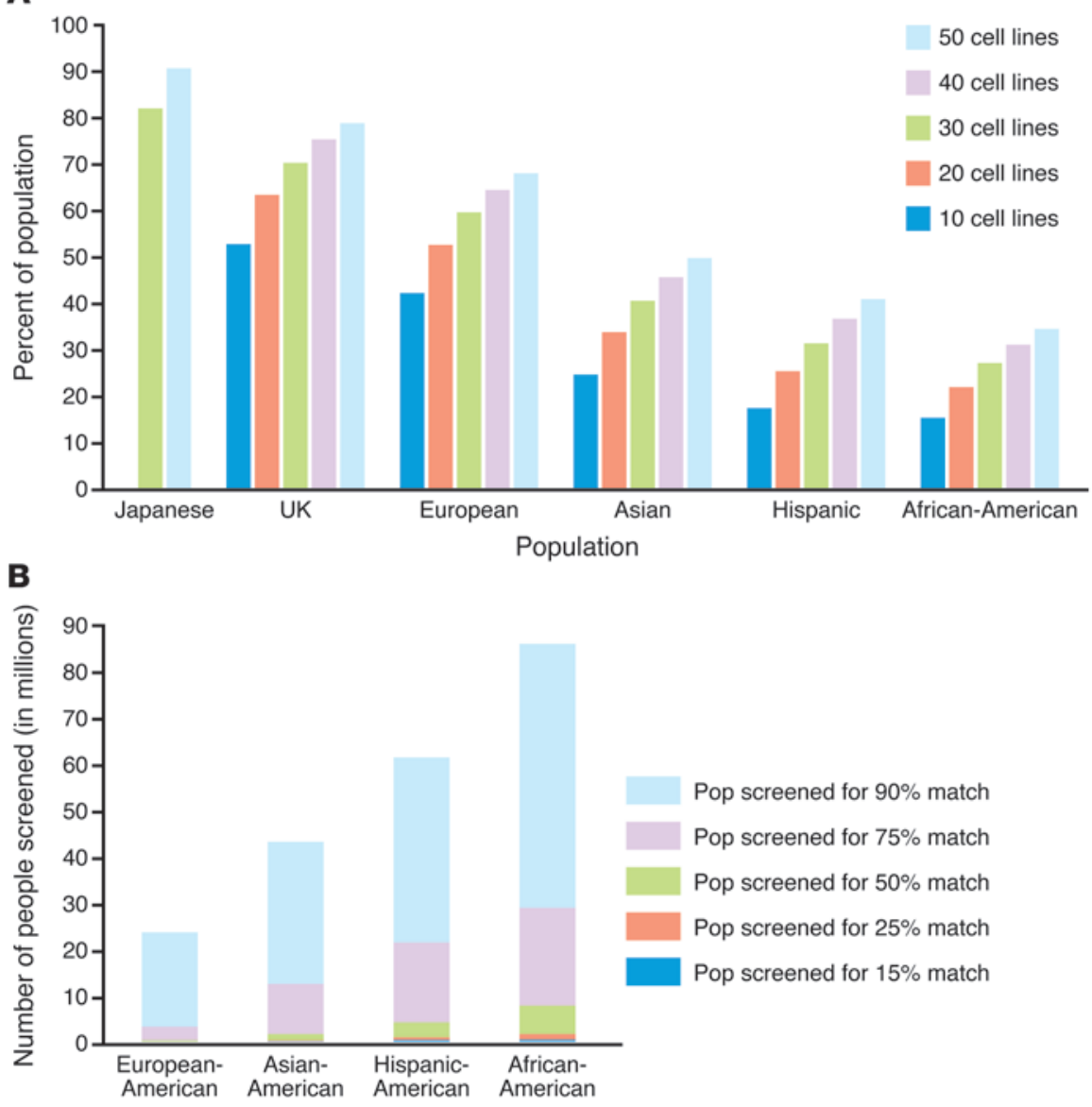

Figure 2. Considerations for iPSC biobanking. (A) The fraction of cited populations that would theoretically find an HLA haplotype match in an IPSC bank that maintains a certain number of lines. (B) The number of individuals in four different ethnic groups that would have to be screened in a US population to develop a haplotype-matched iPSC bank to offer potential matches to a given fraction of each group. This figure consists of data compiled from papers cited within this Review (31, 34, 35). Pop, population.

HLA-matched human iPSC banks have a number of potential advantages, which include (a) providing researchers with a tissue source to help tailor therapeutic development to address immunogenicity concerns for a specific product, and (b) making HLA-matched and even individualized iPSC products a clinical reality. However, the genetic complexity required of such a bank in order to be useful across ethnic barriers is an important aspect to understand before undertaking such development.

Experience with solid organ and bone marrow transplantation has been used to help estimate the scale of iPSC banking that would be required to provide adequately matched tissues in a population. The number of cell lines theoretically required is highly dependent on the demographics and genetic heterogeneity of a region. On the

Autologous tissues have the advantage of not requiring immunosuppression and are likely exempt from immunologic rejection. Although the recipient can tolerate autologous iPSC transplantation, the high costs involved in autologous therapy compared with allogeneic cell banking are a major concern. In addition, the lack of immune rejection in the autologous setting raises the question of whether the risk of teratoma formation might be heightened, as any residual undifferentiated cells might not be detected by the recipient's immune system. Studies using syngeneic xenotransplantation animal models might shed some light on this issue; however, it would still be very difficult to predict problems with human PSCs based on mouse-to-mouse studies. The rapid advancement of iPSC technology makes these questions immediately relevant, as medical communities are starting to build tissue banks that could offer groundbreaking treatments (42).

\section{Considerations for tissue source development}

There are three options for tissue sourcing when developing iPSC-derived products: (a) autologous iPSCs, (b) allogeneic iPSCs or ESCs, and (c) HLA-matched allogeneic iPSCs. While autologous cells would be developed on an individual patient basis, allogeneic cells could be banked as a single product en masse. Currently, most groups with products nearing or in clinical testing are developing single allogeneic ESC lines, but as scientific understanding of successful tissue transplantation and immune rejection evolve, an HLA-matched allogeneic iPSC tissue bank may prove valuable. more favorable end of the spectrum, Nakajima et al. have shown that relatively small numbers of cell lines can be used to provide adequate coverage to a Japanese population (43). Using 2,578 donors from a hematopoietic stem cell registry, they were able to estimate the HLA-matching rates of human ESC lines from randomly donated embryos. Nakatsuji et al. were able to replicate this study for iPSCs by estimating that screening a database of 24,000 individuals would lead to 50 homozygous cell lines generated to provide a haplotype match for $90.7 \%$ of the Japanese population at $H L A-A, H L A-B$, and $H L A-D R$ loci with two-digit specification (44). To extend these results to four-digit specifications, approximately 160,000 individuals would have to be screened to generate 140 homozygous cell lines for similar coverage (45). These findings were confirmed in subsequent studies, which showed that 150 homozygous cell lines could provide a haplotype match for $93 \%$ of the population of the United Kingdom $(46,47)$.

Other researchers have shown that generating a master cell bank for more diverse populations would be far more challenging (48). Gourraud et al. used a probabilistic model to estimate the rate of haplotype matching of a carefully selected homozygous HLA-type iPSC bank for a North American population (48). Their work demonstrates disparities for screening a diverse population. For example, they estimate that 22,000 individuals of European descent would have to be screened to generate 17 iPSC lines to offer a haplotype match to approximately $50 \%$ of that patient population. A screen of 100,000 random individuals in 
the same North American population would only offer a haplotype match to $45 \%$ of Hispanics, $35 \%$ of Asian Americans, and $22 \%$ of African Americans (Figure 2). Similarly, an iPSC bank of the 100 most common HLA types population wide would offer a haplotype match to $78 \%$ of individuals of European descent, $63 \%$ of Asians, $52 \%$ of Hispanics, and $45 \%$ of African Americans (48). These results suggest that customized banking for each ethnic group does not necessarily solve this problem. An iPSC bank of homozygous cell lines containing the 20 most common HLA types in an ethnic population could be built by screening 26,000 individuals of European descent but would require screening of 110,000 African Americans. This bank would offer a haplotype match for $>50 \%$ of individuals of European heritage but only approximately $22 \%$ of African Americans. Hence, an allogeneic cell bank in genetically homogenous countries like Japan or Iceland could be a more viable option, whereas a similar bank in the US may be cost prohibitive.

There are also risks for health delivery disparities that must be considered, because groups of lower socioeconomic standing might require more extensive outreach in terms of tissue donation and storage, to offer treatment options to a significant portion of the population. Tissue storage is also complicated for groups with rare haplotypes, as regulations prohibit use of tissues stored for longer than five years (49). Finally, it should be noted that even highly matched cells could still trigger rejection. A recent study showed that ESCs made via nuclear transfer are still rejected when transplanted into the nuclear donor, presumably because of differences in mitochondrial antigens alone (50). Accordingly, some degree of immunosuppression may be required, even in highly homogeneous populations, although this remains speculative.

\section{CGMP requirements for HLA-matched allogeneic cell banking}

As described above, the scale of iPSC banking necessary to provide tissue for a diverse population is large. While start-up costs and time investment would be high, there are potential production benefits to banking allogeneic tissue over an autologous product approach. The most cost-prohibitive component of autologous, allogeneic, and HLA-matched allogeneic cell banking for clinical application is compliance with cGMP standards. cGMP standards require that the product be consistent in both safety and efficacy, that it be screened for the presence of certain pathogens, and that it be "well characterized" in order to exclude any contaminants. While PSC products are subject to existing FDA regulations and do not constitute a new class of biologic products, pioneering studies using human ESCderived therapies (e.g., Geron's spinal cord injury trial, Advanced Cell Technology's macular degeneration trial, and ViaCyte's type 1 diabetes trial; refs. 9, 10,13) have helped to establish a preclinical and clinical development pathway for these products.

A primary concern for autologous iPSC-derived tissue generation is that variability among different stem cell lines can be significant, making the establishment of standard production processes that satisfy FDA regulatory requirements difficult (49). Autologous stem cell-derived tissues could be particularly difficult to standardize. Differences in the donor cell sources, collection methods, and subsequent reprogramming techniques among various laboratories all introduce significant product variability. Product variability can create safety concerns and may delay therapeutic production for conditions that require time-sensitive treatment (e.g., acute myocardial infarction or cerebral vascular accident). An HLA-matched allogeneic cell bank would help alleviate some of these concerns as production and tissue source could be standardized $(49,51)$.

\section{Challenges of iPSC versatility and the need for a reference product}

iPSCs have inherent genetic and epigenetic variations that have been shown to exist among different iPSC lines and among different passages of the same iPSC line as well as between iPSC and ESC lines (52). This can lead to a natural variability in cellular purity and yield in the final product. Existing biologic therapies that have similar problems use reference materials to determine meaningful versus acceptable product variability. For example, small molecules like erythropoietin can be compared to quality -assured reference stock. This will be more difficult with iPSCs, because meaningful genetic mutations, phenotypic variability, and differences in culture purity are poorly understood. Extensive safety testing of every iPSC line of a large HLA-matched bank to determine safety would be costly. For this reason, allogeneic cell banking would allow easier development of reference materials to improve product safety for iPSC product development.

\section{Commercially viable delivery process of cell products}

For regulatory reasons, the speed of clinical delivery may be dramatically increased by an allogeneic cell bank. Regional trade restrictions on biologics could limit clinical delivery of iPSCbased technology if hurdles to distribution are not anticipated. For example, countries such as Brazil and Sweden do not permit the sale of human-derived tissues, products containing genetic material may not be shipped out of India, and many different countries have varying barriers for stem cell-based technologies (53). An allogeneic cell bank could more easily implement production standards that satisfy diverse regulatory requirements and ethical standards simultaneously.

\section{Commercialization process of autologous, allogeneic, and HLA-matched allogeneic therapies} The standardization of production methods and regulatory licensure has business implications that could attract additional investment as well as innovation to stem cell technologies. Currently, there are two "valleys of death" that are well described in the life cycle of technologies making their way from the lab to clinical application. These two valleys are largely shaped by the costs and risks in translating novel therapies from the lab to the preclinical development phase and, ultimately, to the clinical development phase. An allogeneic stem cell bank could offer an attractive source of PSCs on which to build innovative technologies (54). In the first "valley of death," companies may be reluctant to invest in stem cell technology if the product derivative has not been scientifically validated. In the second "valley of death," companies may be averse to initiating clinical trials without fully comprehending production and licensing costs or validating product safety profiles. Hence, institutions or agencies such as 
the California Institute for Regenerative Medicine (CIRM) provide valuable impetus to the field, as they invest in initial developmental efforts, especially in this era of tight federal budget constraints (55). However, given that funding sources like CIRM are impermanent and intended mostly to launch stem cell technologies, an allogeneic cell bank could be a durable means for mitigating further development costs for future therapies.

\section{Cost of developing a clinical product distribution bank}

One of the primary obstacles to the development of iPSC-based therapies is the high production cost. Generating an HLAallogeneic cell bank based on iPSCs is labor intensive, which drives the majority of the cost and makes the cost of production difficult to reduce. We estimate that each line requires approximately two to four months to develop, starting from collection of primary cells for reprogramming to expansion of a meaningful iPSC population. Based on our experience, for an HLAmatched iPSC bank, companies should aim to store approximately 200 to 300 vials at approximately $2 \times 10^{6}$ cells per vial. This should represent a sufficient number of PSCs from which a master and working banks of lines intended to produce allogeneic products can be established. Excluding high start-up costs, each line costs approximately $\$ 10,000$ to $\$ 20,000$ to produce and validate (49). The development of products that meet cGMP requirements can increase this cost to $\$ 50,000$ to $\$ 100,000$ per line (56). Development costs are even higher $(\sim 800,000)$ for generating an iPSC-derived tissue product that is suitable for clinical use (49). Although cell product development from autologous sources may be more cost effective in the short run based on these estimates, production of autologous tissues is not as easily scalable. By comparison, while start-up costs for an allogeneic cell bank are higher, there are also many opportunities for cost savings downstream. A sizable fraction of the development cost comes from cGMP requirements. For example, in addition to genetic testing, it is necessary to screen for many infectious agents, including mycoplasma, intracellular bacteria, and viral contaminants (57). Some of the screenings require cultures as long as 28 days and transmission electron microscopy for testing. Nonspecific viral contaminant screening uses embryonated chicken eggs and costs $\sim \$ 12,000$ per cell line. Devito et al. have shown that samples from a single donor of human ESCs can be batched to reliably screen for infectious agents (57). They estimate that a bank pooled from 100 donors with three cell lines per donor could save approximately $\$ 18$ million dollars in the clinical validation process. Although these techniques have yet to receive FDA approval, in the future they could be appropriately developed, scaled, and employed in an allogeneic iPSC bank to offset costs, whereas autologous production would require individualized screening.

\section{FDA regulatory pathway for stem cell-based therapies}

Once all the preclinical and commercialization hurdles have been cleared, investigators or companies have to submit their new stem cell-derived therapy portfolio to the regulatory agencies for independent review and approval. The European Medicines Agency in Europe and the FDA in the US have jurisdiction over the commercialization of stem cell therapies in their respective territories. As discussed earlier, stem cell-based products are considered highly processed biologic tissues and require the submission of an IND application to the FDA prior to embarking on any clinical studies. The goal of these agencies is to review and provide oversight of the stem cell-based clinical trials to ensure their safety and efficacy, and to that end, the studies submitted to the FDA must demonstrate data with scientific merit and credibility. The best practice is to approach and establish a dialogue with the FDA early in the process in order to achieve consensus in the development of criteria for the cell products. An optimal execution of the required deliverables that fulfills safety and efficacy goals will ensure that the product is brought to the market in a timely manner.

\section{Conclusion}

Ongoing advances in stem cell therapeutics have the potential to radically improve current treatment approaches for a variety of diseases. Stem cell researchers who are trying to bring cell therapies to the clinic face significant translational challenges that by necessity involve a long and costly process. The FDA and other agencies have substantial regulatory requirements that stem cell therapies must meet. These include carrying out biodistribution, immunogenicity, tumorigenicity, dose toxicity, and pharmacodynamic proofof-concept studies. To fully realize the tremendous potential of stem cell therapies, careful planning and proper resources must be devoted to meeting regulatory and scientific requirements alike to clearly demonstrate their safety and efficacy.

\section{Acknowledgments}

This work was supported by the American Heart Association (12POST12050363 and 14GNT18970018 to E. Neofytou); NIH grants R01 HL123968 and NIH R24 HL117756 (to J.C. Wu); and the California Institute for Regenerative Medicine (IT1-06596, TR3-05556, and DR2A-05394 to J.C. Wu).

Address correspondence to: Joseph C. Wu, 265 Campus Drive, G1120B, Stanford, California 94305-5454, USA. Phone: 650.736.2246; E-mail: joewu@stanford.edu.
1. Eming SA, Martin P, Tomic-Canic M. Wound repair and regeneration: Mechanisms, signaling, and translation. Sci Transl Med. 2014;6(265):265sr6.

2. Thomson JA, et al. Embryonic stem cell lines derived from human blastocysts. Science. 1998;282(5391):1145-1147.

3. Hyun I. The bioethics of stem cell research and therapy. JClin Invest. 2010;120(1):71-75.
4. Zhan X, et al. Functional antigen-presenting leucocytes derived from human embryonic stem cells in vitro. Lancet. 2004;364(9429):163-171.

5. Takahashi K, Yamanaka S. Induction of pluripotent stem cells from mouse embryonic and adult fibroblast cultures by defined factors. Cell. 2006;126(4):663-676.

6. Takahashi K, et al. Induction of pluripotent stem cells from adult human fibroblasts by defined factors. Cell. 2007;131(5):861-872.

7. Matsa E, Burridge PW, Wu JC. Human stem cells for modeling heart disease and for drug discovery. Sci Transl Med. 2014;6(239):239ps6.

8. Scott CT, Magnus D. Wrongful termination: lessons from the geron clinical trial. Stem Cells Transl Med. 2014;3(12):1398-1401.

9. Lebkowski J. GRNOPC1: the world's first embryonic stem cell-derived therapy. Interview with 
Jane Lebkowski. Regen Med. 2011;6(6 suppl):11-13.

10. Schwartz SD, et al. Human embryonic stem cell-derived retinal pigment epithelium in patients with age-related macular degeneration and Stargardt's macular dystrophy: follow-up of two open-label phase $1 / 2$ studies. Lancet. 2015;385(9967):509-516.

11. Schwartz SD, et al. Embryonic stem cell trials for macular degeneration: a preliminary report. Lancet. 2012;379(9817):713-720.

12. Cyranoski D. Next-generation stem cells cleared for human trial. Nature News. http://www.nature. com/news/next-generation-stem-cells-clearedfor-human-trial-1.15897. Updated September 12, 2014. Accessed May 5, 2015.

13. [No authors listed]. First stem cell-derived islets in humans. Nat Biotech. 2014;32(10):969.

14. Menasche P, et al. Towards a clinical use of human embryonic stem cell-derived cardiac progenitors: a translational experience. Eur Heart $J$. 2015;36(12):743-750.

15. Garber K. Inducing translation. Nat Biotechnol. 2013;31(6):483-486.

16. Pearl JI, Kean LS, Davis MM, Wu JC. Pluripotent stem cells: immune to the immune system? Sci Transl Med. 2012;4(164):164ps25.

17. Carpenter MK, Frey-Vasconcells J, Rao MS. Developing safe therapies from human pluripotent stem cells. Nat Biotechnol. 2009;27(7):606-613.

18. Feigal EG, Tsokas K, Viswanathan S, Zhang J, Priest C, Pearce J, Mount N. Proceedings: international regulatory considerations on development pathways for cell therapies. Stem Cells Transl Med. 2014;3(8):879-887.

19. Bosse R, Singhofer-Wowra M, Rosenthal F, Schulz G. Good manufacturing practice production of human stem cells for somatic cell and gene therapy. Stem Cells. 1997; 15(suppl 1):275-280.

20. Bosse R, et al. Production of stem-cell transplants according to good manufacturing practice. Ann Hematol. 2000;79(9):469-476.

21. Cao N, et al. Highly efficient induction and long-term maintenance of multipotent cardiovascular progenitors from human pluripotent stem cells under defined conditions. Cell Res. 2013;23(9):1119-1132.

22. Burridge PW, et al. Chemically defined generation of human cardiomyocytes. Nat Methods. 2014;11(8):855-860.

23. Griessinger $\mathrm{CM}$, et al. $64 \mathrm{Cu}$ antibody-targeting of the T-cell receptor subsequent internalization enables in vivo tracking of lymphocytes by PET. Proc Natl Acad Sci U S A. 2015;112(4):1161-1166.

24. Nguyen PK, Riegler J, Wu JC. Stem cell imaging: from bench to bedside. Cell Stem Cell. 2014;14(4):431-444.

25. Li Z, et al. Comparison of reporter gene and iron particle labeling for tracking fate of human embryonic stem cells and differentiated endothelial cells in living subjects. Stem Cells.
2008;26(4):864-873.

26. Lee AS, et al. Preclinical derivation and imaging of autologously transplanted canine induced pluripotent stem cells. J Biol Chem. 2011;286(37):32697-32704.

27. Cunningham JJ, Ulbright TM, Pera MF, Looijenga LH. Lessons from human teratomas to guide development of safe stem cell therapies. Nat Biotechnol. 2012;30(9):849-857.

28. Lee AS, Tang C, Rao MS, Weissman IL, Wu JC. Tumorigenicity as a clinical hurdle for pluripotent stem cell therapies. Nat Med. 2013;19(8):998-1004.

29. Toupet K, et al. Long-term detection of human adipose-derived mesenchymal stem cells after intraarticular injection in SCID mice. Arthritis Rheum. 2013;65(7):1786-1794.

30. Laflamme MA, et al. Cardiomyocytes derived from human embryonic stem cells in pro-survival factors enhance function of infarcted rat hearts. Nat Biotechnol. 2007;25(9):1015-1024.

31. Wiewrodt R, et al. Adenovirus-mediated gene transfer of enhanced Herpes simplex virus thymidine kinase mutants improves prodrugmediated tumor cell killing. Cancer Gene Ther. 2003;10(5):353-364.

32. Kooreman NG, Ransohoff JD, Wu JC. Tracking gene and cell fate for therapeutic gain. Nat Mater 2014;13(2):106-109.

33. Cao F, et al. In vivo visualization of embryonic stem cell survival, proliferation, and migration after cardiac delivery. Circulation. 2006;113(7):1005-1014.

34. Ben-David U, et al. Selective elimination of human pluripotent stem cells by an oleate synthesis inhibitor discovered in a high-throughput screen. Cell Stem Cell. 2013;12(2):167-179.

35. Lee MO, et al. Inhibition of pluripotent stem cell-derived teratoma formation by small molecules. Proc Natl Acad Sci U S A. 2013;110(35):E3281-E3290.

36. Viswanathan S, Rao M, Keating A, Srivastava A. Overcoming challenges to initiating cell therapy clinical trials in rapidly developing countries: India as a model. Stem Cells Transl Med. 2013;2(8):607-613.

37. de Almeida PE, Ransohoff JD, Nahid A, Wu JC. Immunogenicity of pluripotent stem cells and their derivatives. Circ Res. 2013;112(3):549-561.

38. Koch CA, Geraldes P, Platt JL. Immunosuppression by embryonic stem cells. Stem Cells. 2008;26(1):89-98.

39. Drukker M, et al. Characterization of the expression of MHC proteins in human embryonic stem cells. Proc Natl Acad Sci U S A. 2002;99(15):9864-9869.

40. Zhao T, Zhang ZN, Rong Z, Xu Y. Immunogenicity of induced pluripotent stem cells. Nature. 2011;474(7350):212-215.

41. de Almeida PE, et al. Transplanted terminally differentiated induced pluripotent stem cells are accepted by immune mechanisms similar to self- tolerance. Nat Commun. 2014;5:3903.

42. Marx V. Stem cells: disease models that show and tell. Nat Methods. 2015;12(2):111-114.

43. Nakajima F, Tokunaga K, Nakatsuji N. Human leukocyte antigen matching estimations in a hypothetical bank of human embryonic stem cell lines in the Japanese population for use in cell transplantation therapy. Stem Cells. 2007;25(4):983-985.

44. Nakatsuji N, Nakajima F, Tokunaga K. HLA-haplotype banking and iPS cells. Nat Biotechnol. 2008;26(7):739-740.

45. Okita K, et al. A more efficient method to generate integration-free human iPS cells. Nat Methods. 2011;8(5):409-412.

46. Taylor CJ, Bolton EM, Pocock S, Sharples LD, Pedersen RA, Bradley JA. Banking on human embryonic stem cells: estimating the number of donor cell lines needed for HLA matching. Lancet. 2005;366(9502):2019-2025.

47. Taylor CJ, Peacock S, Chaudhry AN, Bradley JA, Bolton EM. Generating an iPSC bank for HLAmatched tissue transplantation based on known donor and recipient HLA types. Cell Stem Cell. 2012;11(2):147-152.

48. Gourraud PA, Gilson L, Girard M, Peschanski M. The role of human leukocyte antigen matching in the development of multiethnic "haplobank" of induced pluripotent stem cell lines. Stem Cells. 2012;30(2):180-186.

49. Bravery CA. Do human leukocyte antigen-typed cellular therapeutics based on induced pluripotent stem cells make commercial sense? Stem Cells Dev. 2015;24(1):1-10.

50. Deuse T, et al. SCNT-derived ESCs with mismatched mitochondria trigger an immune response in allogeneic hosts. Cell Stem Cell. 2015;16(1):33-38

51. Prescott $C$. The business of exploiting induced pluripotent stem cells. Philos Trans $R$ Soc Lond B Biol Sci. 2011;366(1575):2323-2328.

52. Liang G, Zhang Y. Embryonic stem cell and induced pluripotent stem cell: an epigenetic perspective. Cell Res. 2013;23(1):49-69.

53. Andrews PW, et al. Harmonizing standards for producing clinical-grade therapies from pluripotent stem cells. Nat Biotechnol. 2014;32(8):724-726.

54. Thompson SD. Scientific innovation's two Valleys of Death: how blood and tissue banks can help to bridge the gap. Stem Cells Dev. 2014;23(suppl 1):68-72.

55. Trounson A, DeWitt ND, Feigal EG. The Alpha Stem Cell Clinic: a model for evaluating and delivering stem cell-based therapies. Stem Cells Transl Med. 2012;1(1):9-14.

56. Jacquet L, et al. Strategy for the creation of clinical grade hESC line banks that HLA-match a target population. EMBO Mol Med. 2013;5(1):10-17.

57. Devito L, et al. Cost-effective master cell bank validation of multiple clinical-grade human pluripotent stem cell lines from a single donor. Stem Cells Transl Med. 2014;3(10):1116-1124. 